University of Nebraska - Lincoln

DigitalCommons@University of Nebraska - Lincoln

September 1990

\title{
THE LONG-PERIOD FIELD RR LYRAE STARS
}

\author{
Edward G. Schmidt \\ University of Nebraska-Lincoln, eschmidt1@unl.edu \\ Charles G. Loomis \\ University of Nebraska - Lincoln \\ Andrew T. Groebner \\ University of Nebraska - Lincoln \\ Chris T. Potter \\ University of Nebraska - Lincoln
}

Follow this and additional works at: https://digitalcommons.unl.edu/physicsschmidt

Part of the Physics Commons

Schmidt, Edward G.; Loomis, Charles G.; Groebner, Andrew T.; and Potter, Chris T., "THE LONG-PERIOD FIELD RR LYRAE STARS" (1990). Edward Schmidt Publications. 27.

https://digitalcommons.unl.edu/physicsschmidt/27

This Article is brought to you for free and open access by the Research Papers in Physics and Astronomy at DigitalCommons@University of Nebraska - Lincoln. It has been accepted for inclusion in Edward Schmidt Publications by an authorized administrator of DigitalCommons@University of Nebraska - Lincoln. 


\title{
THE LONG-PERIOD FIELD RR LYRAE STARS
}

\author{
Edward G. Schmidt, Charles G. Loomis, Andrew T. Groebner, and Chris T. Potter \\ Behlen Observatory, Department of Physics and Astronomy, University of Nebraska-Lincoln \\ Received 1989 November 29; accepted 1990 March 12
}

\begin{abstract}
$V R$ light curves have been obtained for 16 faint variable stars with periods shorter than 1 day. Although, as expected, most of the light curves are typical of Bailey-type $a b$ R R Lyrae stars, some have slower rises to maximum and flatter tops. Fourier decomposition was performed to further explore these trends. Combining these data with previously published. Fourier decompositions of RR Lyrae light curves, we find that the Fourier coefficients are essentially constant for periods shorter than three-quarters of a day for Bailey-type $a b$ RR Lyrae stars but that the amplitude ratios, $R(12), R(13)$, and $R(14)$, decrease and the phase differences, $\phi(21)$ and $\phi(31)$, increase at longer periods.

This photometry lends support to the previous suggestion that the star XZ Cet is a good candidate for a Galactic anomalous Cepheid, and we add BW Com as another possibility. When the Fourier parameters for known anomalous Cepheids are compared with those of the RR Lyrae stars, it is found that the scatter precludes an unambiguous segregation of the anomalous Cepheids from the RR Lyrae stars. The longest period star in our sample, V742 Cyg (period $=0.94$ days) is possibly a type II Cepheid but an unambiguous conclusion does not seem possible from Fourier decomposition.
\end{abstract}

Subject headings: stars: Cepheids - stars: pulsation - stars: RR Lyrae

\section{INTRODUCTION}

Among the stars in the fourth edition of the General Catalogue of Variable Stars (Kholopov 1985, 1987; hereafter referred to as the GCVS), pulsating variables in the period range from about three-quarters of a day to slightly more than a day are considerably less common than those of either longer or shorter period. The known examples are mostly faint and have been little studied. Nonetheless, there is reason to believe that interesting stars are to be found in that region. These may include the longest period RR Lyrae stars, the shortest period type II Cepheids (especially if some of them are overtone pulsators), and galactic anomalous Cepheids if any exist.

Simon and Teays (1982) analyzed the light curves of field RR Lyrae stars using Fourier decomposition. The Bailey-type $c$ stars separated clearly from the type $a b$ stars in the Fourier diagrams. There was little evidence of changes in the Fourier parameters for the type $a b$ stars over the range from 0.36 days to 0.75 days. However, the longest period star in the sample, $\mathrm{XZ}$ Cet, stood out; it had Fourier parameters similar to the shorter period type $c$ stars. An examination of the light curve shows that, like those of type $c$ stars, it is sinusoidal in appearance. As a possible explanation, Simon and Teays suggested a period resonance among the longest period type $a b \mathrm{RR}$ Lyrae stars. XZ Cet would then simply be a star which happened to fall very near that resonance. In a subsequent study (Teays and Simon 1985), they obtained the effective temperature from the energy distribution of $\mathrm{XZ}$ Cet and constructed pulsational models. That work cast doubt on the existence of a suitable resonance, and it was suggested that XZ Cet was either a shortperiod, overtone type II Cepheid or an anomalous Cepheid. The modeling favored the latter.

Petersen (1984) carried out Fourier analysis of RR Lyrae stars in $\omega$ Cen. He found that at periods less than about 0.75 days the Fourier coefficients behave similarly to what was found for the field stars. However, at longer periods, not represented in the field star sample, there is a trend for decreasing amplitude ratios and increasing phase differences. However,
$\mathrm{XZ}$ Cet still stands out as peculiar, particularly in the $R(21)$ and $\phi(21)$ diagrams.

In order to clarify the status of XZ Cet, to define the field RR Lyrae sequence in the Fourier diagrams at long period, and to search for other types of stars in this period range, we undertook a program of photometric observations of stars with periods less than a day. Some photoelectric observations were reported by Loomis, Schmidt, and Simon (1988). However, with the photoelectric photometer it was not possible to reach stars fainter than about 13 th magnitude so the sample was very limited. A CCD photometer is now in operation at Behlen Observatory and the sample available is much larger. This paper reports results from that instrument.

\section{OBSERVATIONS}

The observations were all made with the CCD photometer on the $0.7 \mathrm{~m}$ telescope at Behlen Observatory between 1987 June and 1989 April. This device has been described by Schmidt $(1989,1990)$ but a few relevant details will be given here. The photometer employs a TI 4849 CCD which covers an area of the sky of 2.9 by 4.3 with the long axis oriented eastwest. The pixel size, 0 ." 44 , is considerably smaller than typical seeing at Behlen Observatory which has a median value of 2".8 (FWHM). For the observations described here, $V$ and $R$ filters which reproduce the Cousins system were used.

For observations prior to 1988 March, JD 2,447,220, stellar magnitudes were extracted from the CCD frames using a simulated aperture with a diameter of 40 pixels or $17 " .6$. This was chosen to be reasonably close to the aperture size $\left(20^{\prime \prime}\right)$ which was generally necessary with our photoelectric photometer at Behlen Observatory. An aperture optimization algorithm (Schmidt 1990) was used to extract stellar magnitudes for observations beginning in 1988 March. With this algorithm and average seeing about 70 pixels are included in the star aperture. The sky level was taken to be the mean of 768 pixel values within a hollow square region 45 pixels on a side centered on the star image. The determination of the sky level 
makes no appreciable contribution to the error of the photometry when the optimized aperture size is used but was sometimes of importance when the standard aperture size is used.

On all photometric nights, atmospheric extinction was measured and standard stars from the list of Landolt (1983) were observed. As discussed elsewhere (Schmidt 1990), the color terms for this instrument are stable at the 0.024 magnitude level or better over many months. Therefore, average color terms were derived for observations over a period of 1 or 2 months and applied to all the nights in that interval. These monthly mean color coefficients had an rms scatter of 0.018 magnitudes for $V$ and 0.030 magnitudes for $R$ over an interval of 14 months. For nonphotometric nights, color-corrected differential magnitudes were obtained, while on photometric nights standard magnitudes and colors were derived for the comparison stars.

For each variable in this program, at least one comparison star was available within the field of the camera. (This was true of most of the candidate stars. In a few cases no suitable comparison could be found and the variable was dropped from the program.) These comparison stars are identified in Table 1 by their $X$ and $Y$ coordinates (in arcseconds, positive to the north and east) relative to the variable. The magnitudes and colors are means from the number of nights indicated in column (7). Since the choice of comparison stars in a field is limited, good color matching with the variables is not generally possible. The color difference between the mean of the comparison stars and the corresponding variable never exceeded 0.6 in $V-R$.

As a worst case, we can assume that the color terms did not actually change over the $2 \mathrm{yr}$ of observation and that the scatter in the monthly mean coefficients is solely due to measurement errors. These errors will introduce rms errors of 0.011 and 0.018 magnitudes into the differential $V$ and $R$ magnitudes for the largest variable-comparison color difference in our sample. Since the amplitudes of the stars in $V-R$ are no greater than 0.2 magnitudes, errors in the color terms can distort the light curves by no more than 0.002 and 0.004 magnitudes between maximum and minimum. These errors are all sufficiently small to be neglected and this justifies the use of mean coefficients.

Since the actual magnitude levels of the stars are of secondary concern in this project, no special effort was made to obtain accurate magnitudes for the comparison stars. Consequently, some were observed on as many as four photometric nights while several were not observed on any photometric night. Thus the quality of the photometry in Table 1 varies from star to star. However, for those stars observed on more than one night (and not marked as uncertain with a colon in the table), the standard deviations of data from a single night were $0.027 \mathrm{mag}, 0.017 \mathrm{mag}$, and $0.022 \mathrm{mag}$ for $V, R$, and $V-R$, respectively. These values are similar to what was obtained with the photoelectric photometer at Behlen Observatory in the past except for $V-R$. For stars observed on more than one night these standard deviations can be used to estimate the accuracy of the cited magnitudes and colors. As always, values from a single night should be treated as approximate.

Table 2 lists the variables together with the interval during which they were observed, the adopted periods, the epochs of maximum, intensity mean magnitudes, and the number of light curve points obtained. In half of the stars the periods from the GCVS were found to be in error. We redetermined periods for those stars from our data only and these periods are indicated by footnotes in Table 2. The differences between the new
TABLE 1

COMPARISON STAR DATA

\begin{tabular}{|c|c|c|c|c|c|c|}
\hline $\begin{array}{c}\text { Star } \\
(1)\end{array}$ & $\begin{array}{l}X \\
\left({ }^{\prime \prime}\right) \\
(2)\end{array}$ & $\begin{array}{c}Y \\
\left({ }^{\prime \prime}\right) \\
(3)\end{array}$ & $\begin{array}{c}V \\
(\mathrm{mag}) \\
(4)\end{array}$ & $\begin{array}{c}R \\
\text { (mag) } \\
(5)\end{array}$ & $\begin{array}{c}V-R \\
(\mathrm{mag}) \\
(6)\end{array}$ & $\begin{array}{c}n \\
(7)\end{array}$ \\
\hline GM And $\mathrm{C1}^{\mathrm{a}}$ & -134 & -26 & 15.24 & 14.75 & 0.49 & 2 \\
\hline GM And C2 & -93 & 22 & 14.07 & 13.77 & 0.30 & \\
\hline GM And $C 3^{b}$ & 35 & 15 & 15.43 & 15.02 & 0.41 & \\
\hline TY Cam C1 ${ }^{\mathrm{c}}$ & 30 & 15 & 15.61 & 15.10 & 0.51 & 4 \\
\hline TY Cam C2 . & -43 & -15 & 15.52 & 15.10 & 0.42 & \\
\hline TY Cam C3 & 4 & 78 & 15.40 & 14.92 & 0.48 & \\
\hline TY Cam C4 ${ }^{d}$ & -125 & 34 & 14.27 & 13.89 & 0.38 & \\
\hline TY Cam C5 ${ }^{\mathrm{d}}$ & -136 & 81 & 15.31 & 14.86 & 0.45 & \\
\hline BR Cas C1 ......... & -62 & -10 & 12.84 & 12.46 & 0.38 & 2 \\
\hline BR Cas C2 & -95 & -28 & 11.42 & 11.02 & 0.40 & \\
\hline UZ Com C1 & -103 & -112 & 13.66 & 13.25 & 0.41 & 4 \\
\hline BW Com C1 ........ & -114 & 50 & 14.44 & 13.97 & 0.47 & 4 \\
\hline $\mathrm{CY} \operatorname{Com~} \mathrm{Cl}^{\mathrm{e}}$ & 17 & -80 & & & & \\
\hline CV Cyg C1 ${ }^{\mathrm{f}}$ & 73 & -55 & -0.01 & 0.00 & -0.01 & \\
\hline CV Cyg C2 & 41 & -9 & 0.66 & 0.63 & 0.03 & \\
\hline CV Cyg C3 & 21 & -4 & -0.65 & -0.63 & -0.02 & \\
\hline V742 Cyg C1 ${ }^{a}$ & 88 & 66 & 14.44 & 14.05 & 0.39 & 2 \\
\hline V742 Cyg C2 & 29 & 40 & 13.59 & 13.32 & 0.27 & \\
\hline V742 Cyg C3 ....... & -24 & 40 & 13.59 & 13.34 & 0.25 & \\
\hline OX Her C1 $\ldots \ldots \ldots$ & 34 & -26 & 12.98: & 12.20: & 0.78: & 2 \\
\hline OX Her C2 $\ldots$ & -38 & -25 & 13.66: & 13.32: & 0.34: & \\
\hline V692 Her C1 ....... & 79 & 17 & 13.64 & 13.28 & 0.36 & 1 \\
\hline DR Lyr C1 .......... & -44 & -15 & 14.23 & 13.61 & 0.62 & 3 \\
\hline V768 Oph C1 ....... & 113 & -18 & 11.78: & 11.47: & 0.31 & 2 \\
\hline V768 Oph C2 ....... & 58 & 4 & 11.80: & 11.21: & 0.59 : & \\
\hline V768 Oph C3 ${ }^{a} \ldots \ldots$ & -20 & -33 & 14.61: & 14.24: & 0.37: & \\
\hline AN Per C1 ......... & 28 & -37 & 14.51 & 13.80 & 0.71 & 2 \\
\hline AN Per C2 & 83 & 40 & 14.93 & 14.16 & 0.77 & \\
\hline AN Per $\mathrm{C}^{\mathbf{a}} \ldots \ldots \ldots$ & -18 & 65 & 16.34 & 15.58 & 0.76 & \\
\hline AN Per C4 .......... & 84 & -73 & 14.87 & 14.24 & 0.63 & \\
\hline AN Per C5 .......... & -40 & -57 & 13.81 & 13.24 & 0.57 & \\
\hline SX Tri C1 & 61 & 49 & 15.19: & 14.83: & $0.37:$ & 1 \\
\hline SX Tri C2 ........... & -19 & 15 & 14.94: & 14.62: & $0.32:$ & \\
\hline SX Tri C3 .......... & -95 & 59 & 15.44: & 14.90: & $0.54:$ & \\
\hline BX Vir C1 .......... & -65 & -133 & 12.51 & 12.13 & 0.38 & 1 \\
\hline GI Vir C1 $\ldots \ldots \ldots \ldots$ & -25 & 20 & 14.92 & 14.52 & 0.40 & 1 \\
\hline
\end{tabular}

a Not used as a comparison star due to faintness.

${ }^{b}$ GM and C3: Variable. The period cannot be determined from the available data but appears to be approximately 0.215 days. The amplitudes in $V$ and $R$ are about 0.30 mag and 0.37 mag, respectively. Not used as a comparison star.

c TY Cam C1: Excess scatter suggests variability of no more than a few tenths of a magnitude. No period could be derived from the available data. Not used as a comparison star.

d Not used as comparison star because not generally in the frame.

e CY Com: Not observed on any photometric night. The comparison star magnitudes are approximately $V=14.6$ and $R=14.2$.

f CV Cyg: Not observed on any photometric night so differential values are given for the comparison stars. The mean magnitudes of the three comparison stars are approximately $V=13.9$ and $R=13.6$.

periods and the catalog periods were less than 0.003 days for all of the stars exceept CV Cyg where the catalog period of 0.98 days is substantially longer than our value of 0.74 days. Two additional stars, NO Cas and CN Tau, were found to have periods of $\sim 2$ days and are not discussed here as they are clearly not RR Lyrae stars. It is not obvious how many of the period differences are due to errors in the original determinations and how many represent period changes. The epochs of maximum were determined from our observations only. All except two of the stars in Table 2 are classified as RRAB (i.e., Bailey-type $a b$ RR Lyrae stars) in the GCVS. The type for CV Cyg is given as EW/DW (eclipsing binary similar to W UMa). However, this is clearly erroneous from the light curve in Figure 1. The misclassification might have resulted from the 
TABLE 2

\begin{tabular}{|c|c|c|c|c|c|c|}
\hline Star & $\begin{array}{c}\text { Interval } \\
\text { Observed } \\
(\mathrm{JD}-2,440,000)\end{array}$ & $\begin{array}{l}\text { Period } \\
\text { (days) }\end{array}$ & $\begin{array}{c}\text { Epoch } \\
(\mathrm{JD}-2,440,000)\end{array}$ & $\begin{array}{c}\langle V\rangle \\
\text { (mag) }\end{array}$ & $\begin{array}{c}\langle R\rangle \\
\text { (mag) }\end{array}$ & $n$ \\
\hline GM And .......... & $7414-7548$ & 0.7067585 & 7548.628 & 12.98 & 12.72 & 32 \\
\hline TY Cam ............ & $7514-7590$ & 0.670067 & 7590.754 & 13.09 & 12.74 & 41 \\
\hline BR Cas ............ & 7414-7548 & $0.60823^{a}$ & 7548.618 & 13.34 & 12.71 & 35 \\
\hline UZ Com .......... & $7241-7672$ & 0.736941 & 7306.715 & 13.28 & 12.99 & 38 \\
\hline BW Com .......... & $7240-7636$ & $0.7337^{\mathrm{a}}$ & 7322.693 & 14.60 & 14.32 & 35 \\
\hline CY Com ......... & $7569-7625$ & $0.7583^{\mathrm{a}}$ & 7625.64: & b & $\mathrm{b}$ & 17 \\
\hline CV Cyg ........... & $7279-7465$ & $0.7395^{\mathrm{a}}$ & 7368.876 & 0.02 & 0.14 & 38 \\
\hline V742 Cyg ........ & $7041-7467$ & $0.93645^{\mathrm{a}}$ & 7061.613 & 15.85 & 15.13 & 41 \\
\hline OX Her...$\ldots \ldots$ & $7292-7464$ & $0.75748^{\mathrm{a}}$ & 7370.720 & 13.10 & 12.84 & 41 \\
\hline V692 Her ......... & $7292-7319$ & 0.736679 & 7306.873 & 13.97 & 13.66 & 38 \\
\hline DR Lyr $\ldots \ldots \ldots$ & 6999-7443 & 0.8313502 & 7370.760 & 14.36 & 13.98 & 33 \\
\hline V768 Oph ........ & $7322-7667$ & 0.701895 & 7661.866 & 13.72 & 13.40 & 28 \\
\hline AN Per $\ldots \ldots \ldots \ldots$ & $7488-7611$ & $0.6020^{\mathrm{a}}$ & 7591.57: & 14.41 & 13.76 & 32 \\
\hline SX Tri ............ & $7196-7496$ & 0.7429594 & 7464.811 & 14.55 & 14.23 & 16 \\
\hline BX Vir $\ldots \ldots \ldots \ldots$ & $7197-7659$ & $0.8390:^{\mathrm{a}}$ & 7279.851: & b & b & 21 \\
\hline GI Vir ............ & $7245-7629$ & 0.587521 & 7271.647: & 13.93 & 13.66 & 20 \\
\hline
\end{tabular}

a Period different from GCVS.

b Observations do not cover maximum light sufficiently well to determine mean magnitudes.

erroneous period. V742 Cyg is classed in the GCVS as a CWB (type II Cepheid of short period) and we discuss its classification below.

Light curves are plotted in Figure 1. The individual light curve points are available from the first author by electronic mail or by sending a $5 \frac{1}{2}$ inch PC diskette.

Photometric errors arise from a number of sources including readout noise in the detector, photon statistics, imperfect flattening of the frames, imperfect repairs of cosmetic defects, and unrecognized stars in the region used to determine the sky background. The total errors were assessed from the scatter among the comparison stars when more than one was present in a field. It was found that the contribution to the rms scatter in differential magnitudes from one star was 0.011 magnitudes for stars brighter than magnitude 13 in either $V$ or $R$. The contribution to the scatter rose to 0.02 magnitudes by magnitude 14 and 0.025 magnitudes by magnitude 15 . Errors in the light curves can be estimated by combining the errors for the variables and for the comparison stars using these values.

\section{FOURIER DECOMPOSITION}

An examination of the light curves in Figure 1 shows that most of the stars are typical Bailey-type $a b$ RR Lyrae stars as expected. However, it is interesting to note that among the longer period stars in our sample there are several which depart from the classical $a b$ light curve. For example, the stars DR Lyr, BW Com, V692 Her, and V742 Cyg as well as V486 Her (from Loomis, Schmidt, and Simon 1988) tend toward flatter maxima and have a shoulder on the rising branch of the light curve. The rising portion of the light curve is slower than typifies the $a b$ stars.

We have fitted Fourier series to those light curves with adequate phase coverage. Fits were made to both the $V$ and the $R$ curves and were generally of seventh order. In Table 3 we list the mean differences in the various Fourier parameters (following the notation of Simon and Teays 1982) between the $V$ and the $R$ fits. The standard deviations of the individual differences about these means and the standard errors of the means (i.e., the standard deviations divided by the square root of the number of differences used in the mean) are also listed.
We take these standard deviations as an indication of the effect of photometric errors on the derived Fourier parameters since the $V$ and $R$ data are statistically independent. The other significant contribution to the fitting errors comes about due to the phase coverage of the data. Since we have only used those curves with good coverage, this is likely to be significantly smaller than the effects of photometric errors. Clearly, these estimates are very rough and the errors of individual Fourier parameters may vary considerably from the numbers given in Table 3.

The color differences in the amplitude ratios $[R(21), R(31)$, and $R(41)]$ are comparable to the standard errors of the means. We conclude that there is no significant difference in these parameters as derived from the two wavelength bands. On the other hand, the differences for the phase parameters $[\phi(21)$, $\phi(31), \phi(41)]$ are several times the standard errors and are therefore significant.

Table 4 lists the Fourier parameters, the scatter about the fitted curves, and the amplitudes for those stars with adequate phase coverage. $R(21), R(31)$, and $R(41)$ are simple means between the values derived from the $V$ and the $R$ light curves. For $\phi(21)$ and $\phi(31)$ the values from the $R$ light curves were corrected by the mean differences in Table 3 before being averaged. The scatter about the fitted curves is partially due to inadequacies in the representation of the light curves by a

TABLE 3

Differences between Fourier Parameters from $V$ and

$R$

\begin{tabular}{cccc}
\hline \hline $\begin{array}{c}\text { Parameter } \\
(1)\end{array}$ & $\begin{array}{c}\text { Difference } \\
(2)\end{array}$ & $\begin{array}{c}\text { Standard } \\
\text { Deviation } \\
(3)\end{array}$ & $\begin{array}{c}\text { Standard } \\
\text { Error } \\
(4)\end{array}$ \\
\hline$R(21) \ldots \ldots \ldots \ldots$ & 0.021 & 0.041 & 0.013 \\
$R(31) \ldots \ldots \ldots \ldots$ & 0.024 & 0.063 & 0.020 \\
$R(41) \ldots \ldots \ldots \ldots$ & -0.023 & 0.044 & 0.014 \\
$\phi(21) \ldots \ldots \ldots \ldots$ & -0.118 & 0.105 & 0.033 \\
$\phi(31) \ldots \ldots \ldots \ldots$ & -0.358 & 0.204 & 0.064 \\
$\phi(41) \ldots \ldots \ldots \ldots$ & -0.654 & 0.697 & 0.246 \\
\hline \multicolumn{2}{c}{ a Differences in the sense of $R(V)-R(R)$ and $\phi(V)-\phi(R)}$.
\end{tabular}



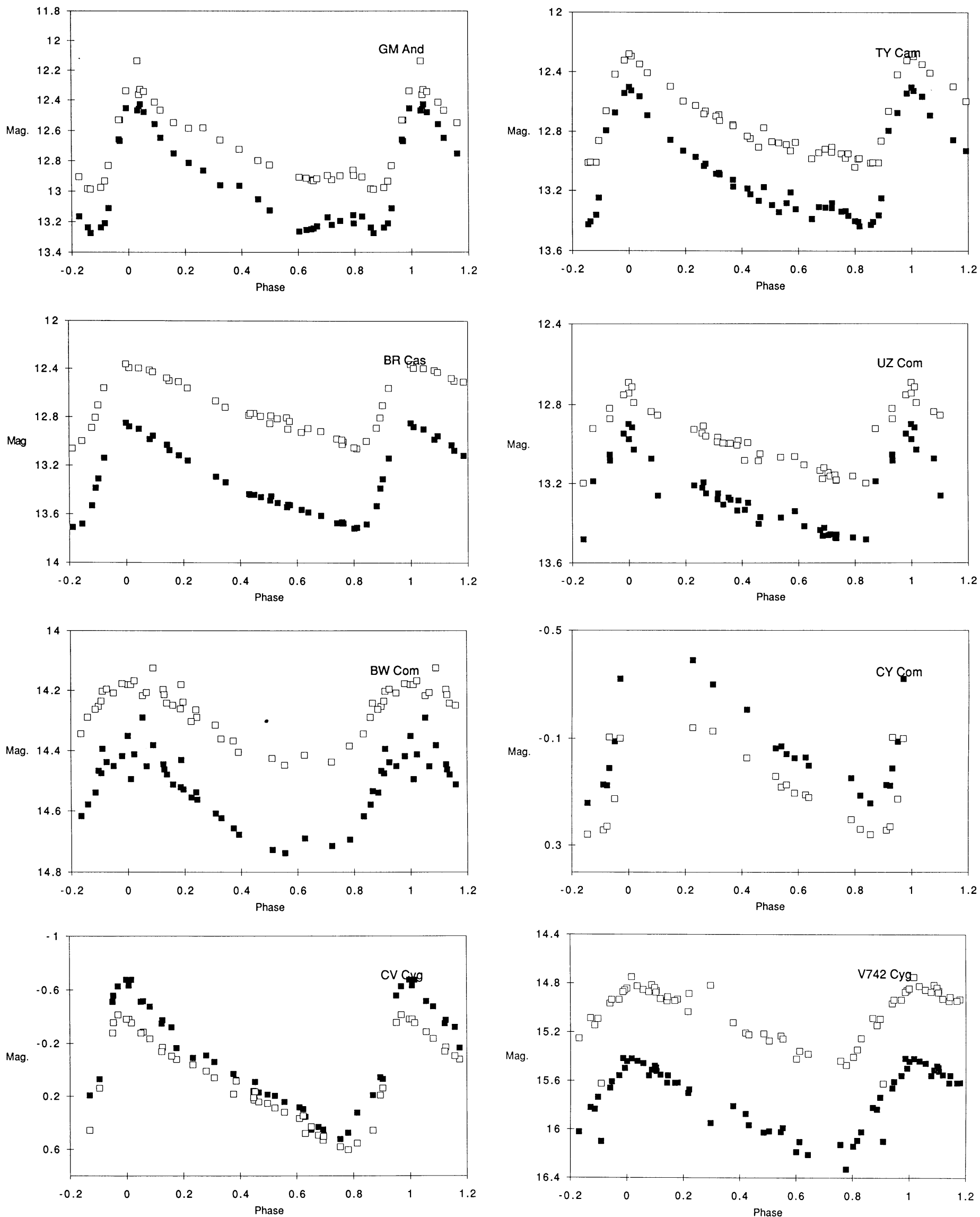

FIG. 1.-Light curves in $V$ and $R$ for RR Lyrae stars. The phases were calculated with the periods and epochs of maximum given in Table 2. Filled squares represent $V$ magnitudes and open squares the $R$ magnitudes. 

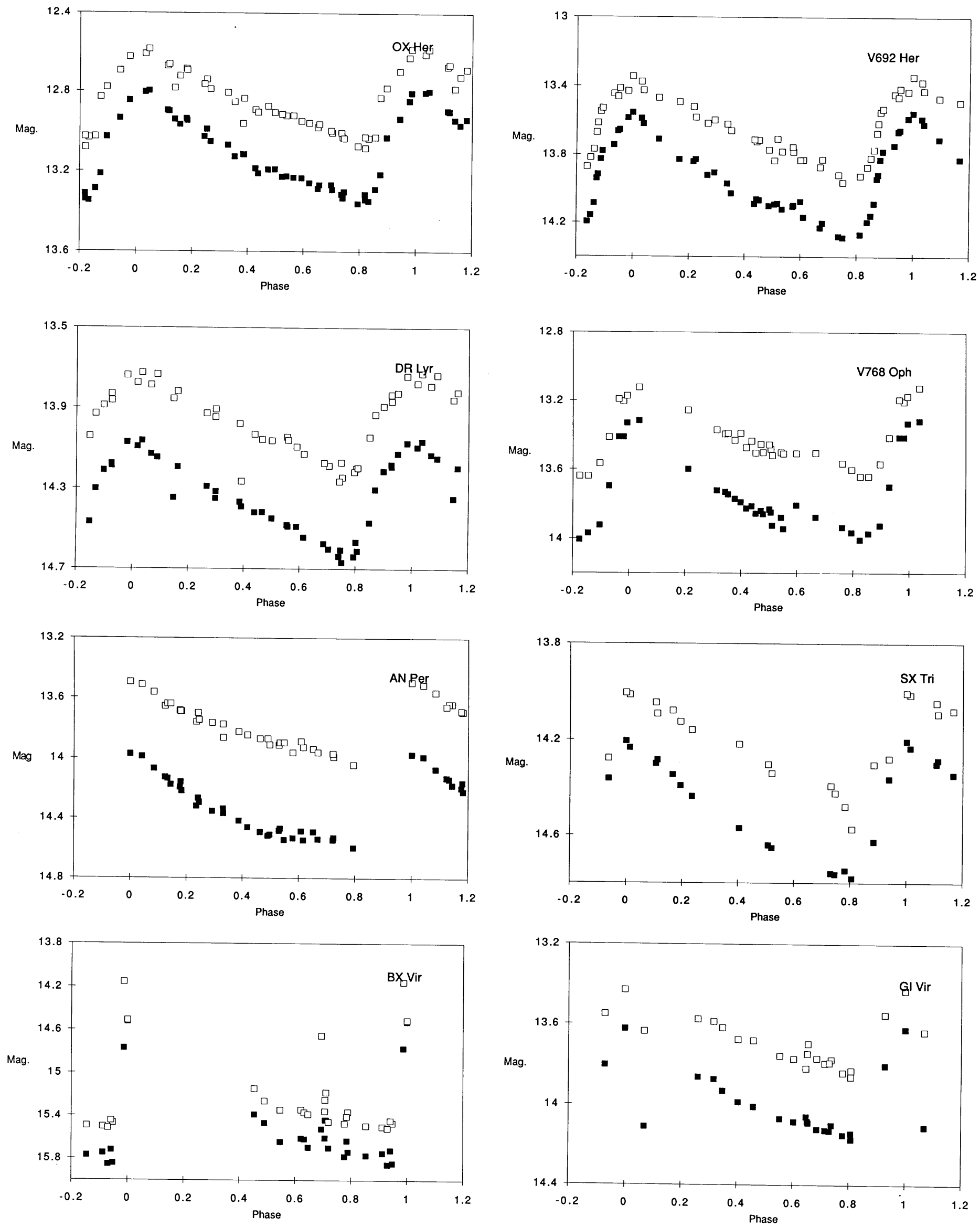

Fig. 1-Continued 
TABLE 4

Light Curve Parameters

\begin{tabular}{|c|c|c|c|c|c|c|c|c|c|}
\hline Star & $R(21)$ & $R(31)$ & $R(41)$ & $\begin{array}{c}\phi \\
(21)\end{array}$ & $\begin{array}{c}\phi \\
(31)\end{array}$ & $\begin{array}{c}\mathrm{S} \\
\mathrm{n} \\
V\end{array}$ & $\begin{array}{l}\text { er } \\
{ }^{\mathrm{a}} \\
R\end{array}$ & $\begin{array}{l}A(V) \\
\text { mag }\end{array}$ & $\begin{array}{l}A(R) \\
\text { mag }\end{array}$ \\
\hline GM And & 0.411 & 0.357 & 0.275 & 4.140 & 1.760 & 19 & 31 & 0.84 & 0.64 \\
\hline TY Cam & 0.486 & 0.330 & 0.224 & 4.006 & 1.865 & 38 & 27 & 0.94 & 0.74 \\
\hline BR Cas ........... & 0.464 & 0.256 & 0.163 & 4.315 & 2.628 & 11 & 19 & 0.86 & 0.70 \\
\hline UZ Com ......... & 0.554 & 0.410 & 0.134 & 4.342 & 2.326 & 35 & 31 & 0.58 & 0.48 \\
\hline BW Com .......... & 0.199 & 0.134 & 0.054 & 3.502 & 1.783 & 33 & 22 & 0.36 & 0.26 \\
\hline CV Cyg ......... & 0.512 & 0.257 & 0.122 & 4.509 & 2.572 & 31 & 25 & 1.20 & 0.01 \\
\hline V742 Cyg ........ & 0.315 & 0.148 & 0.073 & 4.900 & 3.328 & 35 & 39 & 0.82 & 0.64 \\
\hline OX Her $\ldots . . \ldots \ldots$ & 0.454 & 0.254 & 0.125 & 4.239 & 2.608 & 19 & 27 & 0.52 & 0.48 \\
\hline V692 Her ........ & 0.484 & 0.296 & 0.134 & 4.416 & 2.824 & 26 & 27 & 0.74 & 0.58 \\
\hline DR Lyr $\ldots \ldots \ldots$ & 0.446 & 0.166 & 0.108 & 4.546 & 2.990 & 19 & 20 & 0.58 & 0.50 \\
\hline
\end{tabular}

a Scatter of individual data points about the fitted Fourier series.

finite-order Fourier series and partially due to observational error. The values listed for the $V$ fits are comparable to the larger values listed by Simon and Teays. This is reasonable in view of the fact that the stars we have observed are all fainter than the stars they analyzed.

In Figure 2 the Fourier parameters and $V$ amplitudes are plotted against the periods. For comparison these figures also contain similar data for $a b$ RR Lyrae stars from Simon and Teays (1982) and Loomis et al. (1988). The parameters for the Galactic anomalous Cepheid V19 in NGC 5466 (Teays and Simon 1985) and for anomalous Cepheids in the Ursa Minor dwarf galaxy (Nemec 1989) are also shown when available.

\section{DISCUSSION}

The differences between the $V$ and the $R$ Fourier coefficients in Table 3 are similar in size and in the same sense as found previously for classical Cepheids (Simon and Moffett 1985), for type II Cepheids (Petersen and Hansen 1984) and for RR Lyrae stars (Hansen and Petersen 1985). The phase shifts must arise in the stellar atmosphere where differences in the behavior between various spectral regions originate. Their existence shows that detailed theoretical modeling of the Fourier coefficients will require consideration of atmospheric effects. On the other hand, they will possibly serve as useful parameters with which to constrain models in the future.

An examination of Figure 2 shows that the present sample of stars extends the sequences in all the Fourier diagrams toward the location of XZ Cet. In particular, the variables BW Com and V742 Cyg lie quite close to XZ Cet in the amplitude ratio diagrams. However, in no diagram have the new stars actually engulfed XZ Cet. In the $R(21)$ diagram and to a lesser degree in the $R(31)$ and $R(41)$ diagrams a significant gap remains between the bulk of the sample and XZ Cet. The sequences of RR Lyrae stars in the $\phi(21)$ and $\phi(31)$ diagrams appear to curve upward toward the location of XZ Cet at long period but it still lies above any other star. On the other hand, BW Com is well removed from $\mathrm{XZ}$ Cet in the phase diagrams.

A comparison of Figure 2 with the corresponding diagrams for $\omega$ Cen (Petersen 1984, Figs. 4 and 6) shows good agreement except for a small shift in the phase differences attributable to the different spectral band of the magnitudes used. The Petersen amplitude ratio diagrams clearly show decreases in the amplitude ratios at periods longer than about 0.8 days for $R(21)$ and $R(31)$ and longer than 0.7 days for $R(41)$. This is less well defined in our diagrams due to a smaller number of stars but is still present. The increasing phase differences at long period are clearly present in both the field sample and the $\omega$ Cen stars.

If we postulate that the amplitude ratios for the type $a b \mathbf{R} \mathbf{R}$ Lyrae stars do not depend on period shortward of the above limits, the mean values of these parameters are $\langle R(21)\rangle=0.489 \pm 0.048(\sigma),\langle R(31)\rangle=0.311 \pm 0.049$, and $\langle R(41)\rangle=0.187 \pm 0.056$. Thus, the scatter is largely accounted for by the uncertainties estimated above (col. [3] of Table 3). The fact that XZ Cet and BW Com lie between 3.6 and 6.6 standard deviations below the mean in the $R(21)$ diagram and other stars of similar period have $R(21)$ values, in agreement with the short-period stars, is strong evidence that these two stars belong to a different class of variable.

To further clarify the usefulness of the Fourier diagrams in separating RR Lyrae stars from anomalous Cepheids we have plotted the anomalous Cepheids in the Ursa Minor dwarf galaxy (Nemec 1989) and a Galactic anomalous Cepheid, NGC 5466 - V19 (Teays and Simon 1985). Unfortunately, no clear trend emerges, due perhaps in part to poorly determined Fourier coefficients for the faint extragalactic anomalous Cepheids. In the $R(21)$ diagram, four of the anomalous Cepheids fall within the sequence of the Galactic RR Lyrae stars while two of the others are near XZ Cet and BW Com in the lower right corner. It is possible these latter stars and the remaining two anomalous Cepheids form a sequence which descends from the region of the short-period RR Lyrae stars toward the lower right corner of the diagram. In that case, there is no obvious explanation for the anomalous Cepheids which lie in the main band of RR Lyrae stars aside from errors in the coefficients. In the $R(31)$ diagram the situation is similar but there is considerable scatter at the short and long periods which obscures any separation of RR Lyrae stars and anomalous Cepheids. In the $\phi(21)$ and $\phi(31)$ diagrams the anomalous Cepheids fall within the RR Lyrae sequences with a single exception (Ursa Minor-80, $P=0.50$ days).

In summary, it appears from Figure 2 that only in the amplitude ratio diagrams, the $R(21)$ diagram in particular, is there any indication that we can distinguish anomalous Cepheids from RR Lyrae stars and the situation there is confused by the scatter of the anomalous Cepheids. However, several points argue in favor of believing $\mathrm{XZ}$ Cet to be an anomalous Cepheid. There is convincing evidence that NGC 5466-V19 which lies near XZ Cet in both the $R(21)$ and the $\phi(21)$ diagrams is an anomalous Cepheid (Zinn and Dahn 1976; Zinn 

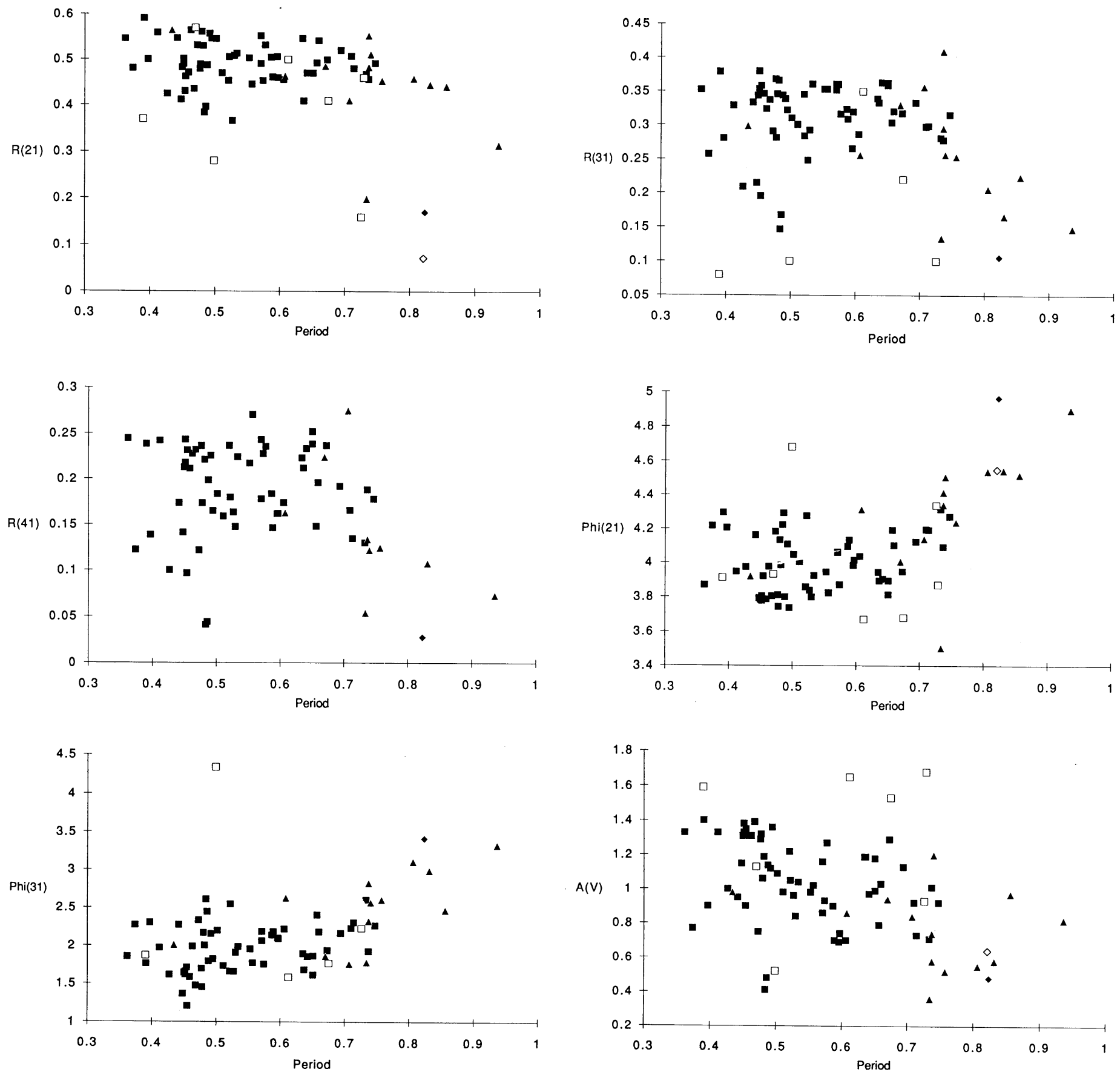

Fig. 2.-Plots of various Fourier parameters and the $V$ amplitude against period for RR Lyrae stars and anomalous Cepheids. The filled squares represent the Bailey type $a b$ RR Lyrae stars from Simon and Teays (1982) except for XZ Cet which is represented by a solid diamond. Triangles denote the stars from Table 4 and from Loomis et al. (1988). Open squares represent anomalous Cepheids in the Ursa Minor dwarf galaxy, while the open diamond represents V19 in NGC 5466.

and King 1982). In addition, the gravity and temperature of XZ Cet is similar to that of NGC 5466-V19 (Teays and Simon 1985) and appropriate for an anomalous Cepheid. There is no other obvious explanation for the difference in light curve shape between the stars in the lower right of the $R(21)$-period diagram and the RR Lyraes at the long-period end of the level sequence. Thus, we continue to regard XZ Cet as a Galactic anomalous Cepheid and add BW Com as another possible example of such a star. V742 Cyg will be considered further below.
Further work is required to substantiate the conclusion of the previous paragraph. In particular, theoretical modeling is required to show whether a sequence of anomalous Cepheids which is separate from the RR Lyrae stars is to be expected in the amplitude ratio diagrams but not in the phase difference diagrams. Models should also be used to show that the constancy of the amplitude ratios with period and the increase in the phase differences at long period have a physical basis. Additional, accurate observations of extragalactic anomalous Cepheids are needed to improve the Fourier parameters for 
those stars and thereby clarify the situation. Finally, a measurement of the absolute magnitudes of XZ Cet and BW Com by the Wesselink method could provide additional evidence of their status. In view of the importance of identifying Galactic anomalous Cepheids to studies of the nature and origin of such stars, these investigations should be undertaken.

It is possible, as mentioned in the Introduction, that our sample contains short-period type II Cepheids. In examining Figure 2, the most likely candidate is V742 Cyg with a period of 0.94 days. This star may very well lie at the end of the RR Lyrae sequences and simply be the longest period RR Lyrae star. On the other hand, it is also possible that the sequences do not curve toward its position sufficiently to encompass it. Again it is the $R(21)$ diagram in which this star is the most separated from the RR Lyrae stars. We can place V742 Cyg on the Fourier diagrams shown by Simon (1986) for short-period type II Cepheids. In doing this we find that V742 Cyg does indeed fall along an extension of the region occupied by the stars denoted by Simon as short-period RRd stars or class $\mathrm{S}$ stars in the $\phi(21)$-period and $\phi(31)$-period diagrams. In the $\phi(21)-\phi(31)$ diagram, V742 Cyg is also among the short-period RR $d$ stars. However, the same could be said of other stars in Table 4. Since they are clearly RR Lyraes, the status of V742
Cyg is ambiguous and we cannot conclude whether it is a long-period RR Lyrae star or a short-period type II Cepheid. A possibility, supported by this discussion, is that the shortperiod type II Cepheids and the RR Lyrae stars form one continuous type II sequence.

To clarify the status of stars like V742 Cyg and to learn the relation between the type II Cepheids and the RR Lyrae stars, we require observations of additional stars with periods near 1 day. Unfortunately, it is difficult to study such stars and there is a serious selection effect against their discovery as variables. To discover and study these stars would require a large-scale project with observations from different parts of the world or observations from an observatory near one of the Earth's poles. Thus, extending this study to longer periods is unlikely in the near future.

The CCD camera system used in this research was obtained through grant AST-8504072 from the National Science Foundation and the work was completed with support from NSF grant AST-8815806. Data reductions and analysis were performed using the facilities of the Minnich Astronomical Computing Center donated by Commander C. B. Minnich.

Hansen, L., and Petersen, J. O. 1985, in IAU Colloquium 82, Cepheids: Theory and Observations, ed. B. Madore (Cambridge: Cambridge University Press, p. 272.

Kholopov, P. N. 1985, General Catalogue of Variable Stars, Fourth Edition, Vol. 1 \& 2, (Moscow: Nauka Publishing House) (GCVS).

Kholopov, P. N. 1987, General Catalogue of Variable Stars, Fourth Edition, Vol. 3 (Moscow: Nauka Publishing House) (GCVS).

Landolt, A. U. 1983, A.J., 88, 439.

Loomis, C., Schmidt, E. G., and Simon, N. R. 1988, M.N.R.A.S., 235, 1059.

Nemec, J. M. 1989, in IAU Colloquium 111, The Use of Pulsating Stars in Fundamental Problems in Astronomy, ed. E. Schmidt (Cambridge: Cambridge University Press), p. 215.

\section{REFERENCES}

Petersen, J. O. 1984, Astr. Ap., 139, 496.

Petersen, J. O., and Hansen, L. 1984, Astr. Ap., 134, 319.

Schmidt, E. G. 1989, in Automatic Small Telescopes, ed. D. S. Hayes and R. M. Genet (Mesa, AZ: Fairborn Press), p. 195.

Schmidt, E. G. 1990, in preparation.

Simon, N. R. 1986, Ap. J., 311, 305.

Simon, N. R., and Moffett, T. J. 1985, Pub. A.S.P., $97,1078$.

Simon, N. R., and Teays, T. J. 1982, Ap. J., 261, 586.

Teays, T. J., and Simon, N. R. 1985, Ap. J., 290, 683.

Zinn, R., and Dahn, C. C. 1976, A.J., 81, 527.

Zinn, R., and King, C. R. 1982, Ap. J., 262, 700.

Andrew T. Groebner and Charles G. Loomis: International Ultraviolet Explorer Observatory, Code 684.9, Goddard Space Flight Center, Greenbelt, MD 20771

Chris T. Potter and Edward G. SChmidt: Department of Physics and Astronomy, University of Nebraska, Lincoln, NE 685880111 